University of Nebraska - Lincoln

DigitalCommons@University of Nebraska - Lincoln

To Improve the Academy

Professional and Organizational Development Network in Higher Education

1994

Do You See What I See?

Karin McGinnis

Kenneth Maeckelbergh

Follow this and additional works at: https://digitalcommons.unl.edu/podimproveacad

Part of the Higher Education Administration Commons

McGinnis, Karin and Maeckelbergh, Kenneth, "Do You See What I See?" (1994). To Improve the Academy. 312.

https://digitalcommons.unl.edu/podimproveacad/312

This Article is brought to you for free and open access by the Professional and Organizational Development Network in Higher Education at DigitalCommons@University of Nebraska - Lincoln. It has been accepted for inclusion in To Improve the Academy by an authorized administrator of DigitalCommons@University of Nebraska - Lincoln. 


\section{Do You See What I See?}

\section{Karin McGinnis and Kenneth Maeckelbergh}

Lakewood Community College

This paper explores the role of visual perception as a value-laden, learned behavior. Through example, including visual conventions, it describes the relationships between perception, culture, and experience as well as the impact visual imagery has in the academic community. Methods for developing critical visual inquiry (visual literacy) are included.

Rarely is visual perception, or what might better be called visual literacy, considered an issue of college curriculum or faculty development. Emphasis is placed upon course content and relevance, teaching and learning method, and the clarity of ideas as expressed in the spoken and the written word, despite the omnipresent use of "visuals." Occasionally there are discussions of values as conveyed through visual imagery but without concern for the "why" of perception. The assumption is that, barring a mechanical problem such as myopia or colorblindness, all individuals see in a similar fashion. But is this really the case? In our culturally diverse society, do you see what I see?

\section{The Importance of Visual Perception}

It is said that $85 \%$ of our knowledge comes through our eyes. Such statements as "I see what you mean" and "seeing is believing" demonstrate the important and accepted correlation between sight and comprehension. How often do you say "oh, just show me," a phrase as grounded in practical reality as "a picture is worth a thousand words." When trying to clarify an idea, how often do you grope for a suitable visual metaphor? Do you expect students and colleagues alike 
to use relevant visual imagery to enhance description? Are illustrations part of lectures and seminars? How often does an image accompany charts and graphs in the pile of overhead transparencies? Are posters, illustrated catalogs, and art works visible in your institution? There is much discussion today about the values imparted by the visual images of television, film, and print. We decry the malevolence of swastikas drawn upon walls, the manipulatory image of "Joe Camel," the negative stereotypes of women, and the omnipresence of sexual imagery. We know that what is seen has impact far beyond some paint on the wall, lines on paper, or staged representations. And yet despite this acknowledgment, there still is little concern for the entire process of visual perception and the origins of the imagery we so easily use. There is a strong assumption that sight is a purely mechanical activity needing no instruction, that given the right physical equipment, we will all see in the same way. And yet, if we really thought this the case, why were so few adults initially concerned about the Camel cigarette advertising? Why do some people see a sexual motif and others a cool role model? Why the concern that the violent images seen on television and in film are the problem, not so much the spoken and written word?

Surely more than mechanics is at work here. It is truly ironic that learning to speak, learning to read, and learning to listen are all important elements of a sound education, whereas learning to see is omitted. We struggle to teach critical thinking and critical reading but not critical seeing. We test for only the gross mechanical aspects of vision, perhaps because they are relatively easy to understand and repair: given the proper lenses or surgery, we assume that all should be able to see. Even color blindness, a physical impairment that can cause social and academic problems, is not always part of standard visual screening. It can and does happen that students enroll in college courses such as art history and chemistry completely unaware that they have a visual disability in the recognition of color.

Beyond such physical problems, all of us, in many everyday situations, know that people do not see in the same way or even see the same thing despite possessing the same visual acuity. All too common are differences in description of vehicles involved in accidents, physical characteristics of burglars, and landmarks along a well-traveled route. Sometimes such divergence is simply a matter of 
inattention, but often it is the result of different ways of seeing. Ample evidence indicates that how we process the visual information presented to our eyes is more than mere mechanics; it is a subjective effort based upon experience, culture, and motivation.

\section{The Process of Visual Perception}

Like all learning, visual perception is experiential. We only understand images through repetition and comparison, developing a visual vocabulary that serves as a constant template in our brains. Once we store a visual image, it can be used as a standard to which we compare any new image. For example, once we have seen and understood the image of a dog, we can draw some conclusions about other four-legged animals, as well as other dogs. Through experience we also establish a scale of visual relationships which enable us to move about with some degree of safety. It is easy to understand such obvious situations as the child who, having never seen a staircase, is unable without instruction to climb it, or the adult from a tropical climate who simply cannot see the ice supporting a car on a frozen Minnesota lake. More difficult to recognize is the cultural language of body movement, spatial relationships, symbols, colors, and shapes. Anger, frustration, and even violence occur because of blindness to these languages. Because images have immediate impact, it is easy to understand the reason for territorial graffiti in inner cities, the handshake of diplomacy, and the finger of insult with which our society is familiar.

Where, when, and how we acquire our visual vocabulary also affects our perception. If learned when very young, an image usually is taken for granted and considered to be the only way something should look. Thus, the first dog a child knows and loves may always be thought of as the one and only ideal dog. If the first dog encountered bites the child , the child may not only fear all similar dogs but actually see them as "all teeth." And a dog seen as quite small by an adult will be seen as large by a small child. Jerome Bruner and Cecile Goodman (Chance, 1989) demonstrated that poverty can affect how children see: given circles to identify as coins the poor children consistently overestimated the sizes. We all have experienced the distorted vision brought on by fatigue, hunger, or anger, as well as conflicts about 
beauty due to unfamiliarity or cultural bias. During the recent American involvement in the Gulf Crisis, the U.S. military had to be taught to both understand and to see the insult implied by exposing the sole of the foot, a gesture with which all Saudis are familiar but which is not even noticed by Americans.

\section{Visual Perception and the Academic Community}

But what about the complex visual vocabulary used throughout the academic world? Is it one of unanimity? Unfortunately, instances of disagreement throughout academe are many, ranging from the obvious, such as protests over poster designs and art works, to the less overt, such as a sense of environmental intimidation. One doesn't need to look beyond the physical surface to find an obvious example of the challenge. Every day students and faculty alike interact in an architectural environment of visual as well as practical significance. From the arrangement of the classroom to the location of student services, an image of the college community is created. The old "halls of ivy" may no longer be the norm, but visual conventions still inhabit the new halls, halls which may even discourage the learning process. For students from the visually complex but intimate environs of the inner city, the sprawling campus in a rural setting may present an image of isolation and dislocation. The columned porticoes of many collegiate buildings may be intimidating to individuals more familiar with classical revival architecture through uncomfortable experiences in courthouses. A large classroom may be seen by some students as a familiar and comfortable space and by others as evidence of the disregard for the individual. How space is organized and used is a direct reflection of social order and cultural conventions. All of the elements of architecture reflect the values of those who create it, values which may or may not enhance the educational atmosphere.

Of equal importance but less obvious are the visual images embedded in course material. In the struggle to inform, we often use illustrations, similes, and visual metaphors to reinforce an idea through an image. Governed by our own experience, we often choose one which is commonly understood. But we also may use one beyond the experience of our audience or one which causes confusion, discom- 
fort, or embarrassment. Often our values are not only conveyed in these visual messages but given power not readily imagined. A common example is the metaphor "run with the ball." It carries the image of an athletic event, for most, of football, a masculine game. Not only does it convey the importance of sports imagery, but it also might be regarded as sexist. Images are both immediately affective and lasting, remembered more strongly than the complete original context and more quickly recalled. Sometimes an image is so interesting, confusing, or disconcerting that it proves more distracting than beneficial and can even completely control the attention, obscuring the context. An image of two men dancing arm in arm is often the only image remembered and queried by students watching a film on the history of the motion picture, obscuring the more important material about process and development of the media.

\section{The Challenge of Visual Perception}

Because of the strength of cultural and personal experience, complete visual objectivity is impossible. Whether in the analysis of beauty, the admiration of specific forms of visual representation, or the role of common images in society, our first approach is through our learned way of seeing. However, all of us can understand the diversity of cultural viewpoints and appreciate the rich complexity of the human experience. Values as revealed in all aspects of the visual world can be considered and respected, given the critical skills. Understanding visual perception and developing visual skills or, what might better be called visual literacy, can be an enlightening and rewarding process, providing connections to other ways of seeing, as well as revealing our own cultural subjectivity. What once may have seemed of little consequence may reveal itself as an exciting possibility-the significance of a gesture so often overlooked, the meaning of a painting once regarded as meaningless, and/or the rich symbolism of an arrangement of objects. We become more aware of the diversity of cultural attitudes toward such familiar visual elements as space, color, and representation. We can analyze visual conventions so common in our lives and question their accuracy or effectiveness, as well as reaffirm their validity. 


\section{Understanding Visual Literacy}

Although experience provides us with one method of comprehending our visual world, art and artists have provided us with the major forms of visual representation. We all possess a visual vocabulary of extreme complexity and flexibility, but it is one framed by artistic tradition and innovation. In order to understand why we see the way we do, one can begin with an analysis of visual convention.

Convention is a form which becomes accepted as the standard representation within a culture, usually because it is so apt and immediate. Americans recognize the "truth" of the sun through the visual representation of a circle with lines radiating outward, even though the sun itself never actually appears in this form. Blue is a color convention for water because natural bodies of it so often reflect blue, not because the water itself is blue in color. Objects distancing in space are represented by diminishing size, not because objects actually shrink with distance, but appear to do so.

Originally developed through an act of creativity, conventions gain their credence through repetition. A crossed band of red ribbon is a recent convention accepted by many to signify support for the victims of AIDS. Not many years ago a yellow ribbon was only a phrase in a song, but today most Americans see it as a symbol of loyalty and reunion. Every day in every aspect of our lives we are directed by the images of visual convention: the "sporty" car, the "power suit," the linear division of space by sidewalks and streets. Human attitudes such as love, anger, fear, and depression can be represented through simple convention. One just needs to look at the comic pages to understand this phenomenon.

Patterns of social organization are reinforced by visual conventions such as the placement and decoration of offices (who has an office with a view and a polished wood desk?), the shape of classrooms, and the arrangement of furniture. So well do we learn to read these visual conventions within our own culture we respond immediately: we drive through an intersection when the light is green; we expect a celebration when we see balloons and colored streamers; and a tall pointed spire we follow to a church. No word need be spoken, and one can, in fact, have quite complicated "visual conversations." 
Unfortunately, by this very ease we are misled into an assumption of visual universality. Even within a culture, conventions often change (at one time a yellow ribbon was a symbol for cowardice), just as they may be completely unrecognized or misunderstood in another culture (linear perspective is not a universal representation of space). The current debate about visual images and stereotypes of Native Americans is one important example of cultural conflict over visual perception.

\section{Developing Visual Literacy}

Our world is filled with far too many images for us to analyze and evaluate each and every one. However, there are practical ways to increase visual literacy:

1. Explore the visual world created by painters, sculptors, and architects. Really look at art and wonder about what you see. Ask others about what they see. Be critical about your response. The accompanying questionnaire can help in this process. By understanding the role and work of visual artists, we can begin to be more sensitive and constructive in our use of imagery.

2. Learn the basic language of the visual arts. Such words as composition, scale, balance, harmony, representation, and expression not only enable us to analyze a work of art, but they also reveal cultural ideas. There are many texts available which define this language.

3. Learn some art history. Even a brief historical survey of art reveals the longevity of standards of representation and the power of images created by artists. Such works as the "Creation of Adam" by Michelangelo, the "Venus de Milo" of ancient Greece, the "American Gothic" of Grant Wood, and the Viet Nam Memorial by Mai Lin have become models, admired and replicated in countless ways. Contemporary American culture reveals values of the Renaissance in Italy as created in the sweet-faced, blond women of Raphael, the powerful males of Michelangelo, and the logical arrangement of space by Alberti. Our symbol of governance, from the dome of the nation's capitol to all the variations in states throughout the country, replicates European ideas and images of an earlier century. Even the white, pointed steeple of New England churches and the desire for a pano- 
ramic view so beloved on calendar illustrations reflect specific cultural attitudes that can be traced through art. By understanding the origins and traditions we can also understand the manipulative power, both positive and negative.

4. Understand visual conventions. Look at printed material and consider the content which is immediately revealed without words. Think about commonly accepted symbols such as the heart, red roses, stars, and crosses. Consider colors which have symbolic meaning.

5. Query others. Ask how they feel about an image, a color, an arrangement of a classroom. Pay attention when you travel and note differences in visual signs, color choice, and arrangement of space. Always look around and think about what you are seeing.

We need to expand our visual vocabulary to be more inclusive, to discard stereotypes or use them in a critical manner, and to make the visual aspect of instruction dynamic and relevant. Before we use an image, we should consider it critically, asking ourselves the following:

1. Why did we choose this image?

2. What is its history in our own lives?

3. Is it reflective of a specific time and culture?

4. Could it be misinterpreted?

5. Would it be understood in another culture?

The same basic questions can be posed in the creation and organization of physical space.

By truly considering visual imagery, one can work toward the positive experience necessary in an academic environment. All visual images can be useful instructional devices when one recognizes the cultural content and poses the question: Do you see what I see? Sensitivity to the diversity of interpretation is a worthwhile enterprise of lasting consequence.

\section{References}

Chance, P. (1989, January). Seeing is believing. Psychology Today, p. 26.

\section{Suggested Reading}

Axtell, R. E. (1991). Gestures: The do's and taboos of body language around the world. New York: John Wiley. 
Berger, A. A. (1984). Signs in Contemporary Culture. Salem, WI: Sheffield.

Berger, J. (1979). Ways of seeing. London: Penguin.

Birren, F. (1963). Color: A survey in words and pictures. New Hyde Park, NY: University Books.

Birren, F. (n.d.). Color psychology and color therapy. New York: McGraw-Hill.

Blonsky, M. (1985). On signs. Baltimore: Johns Hopkins Press.

Buzan, T. (1983). Use both sides of your brain. New York: E. F. Dutton.

Clark, K. (1960). Looking at pictures. New York: Holt, Rinehart \& Winston.

Connerton, P. (1990). How societies remember. New York: Cambridge University Press.

Danto, A. C. (1992). Beyond the Brillo box. New York: Farrar, Strauss, Giraux.

Davis, F. (1974). Inside intuition: What we know about nonverbal communication. New York: McGraw Hill.

Dondis, D. A. (1973). A primer of visual literacy. Cambridge: The MIT Press.

Frisby, J. P. (1980). Seeing: Illusion, brain and mind. Oxford: Oxford University Press.

Gombrich, E. H., Hochberg, J., \& Black, M. (1972). Art, perception, and reality. Baltimore: The Johns Hopkins University Press.

Hall, E. T. (1959). The silent language. Garden City, NY: Doubleday.

Hall, E. T. (1969). The hidden dimension. Garden City, NY: Doubleday.

Hess, E. H. (1975). The tell-tale eye: How your eyes reveal hidden thoughts and emotions. New York: Van Norstrand Reinhold.

King, A. D. (Ed.). (1980). Buildings and society. London: Routledge and Kegan Paul.

Koch, R. (n.d.). The book of signs. New York: Dover Publications.

Lanier, V. (1982). The arts we see: A simplified Introduction to the visual arts. New York: Teachers College Press.

Schrank, J. (1973). Deception detection. Boston: Beacon Press.

Solomon, J. (1988). The signs of our times. Los Angeles: Jeremy P. Tarcher. 


\section{FIGURE 1: Art Criticism Check Sheet}

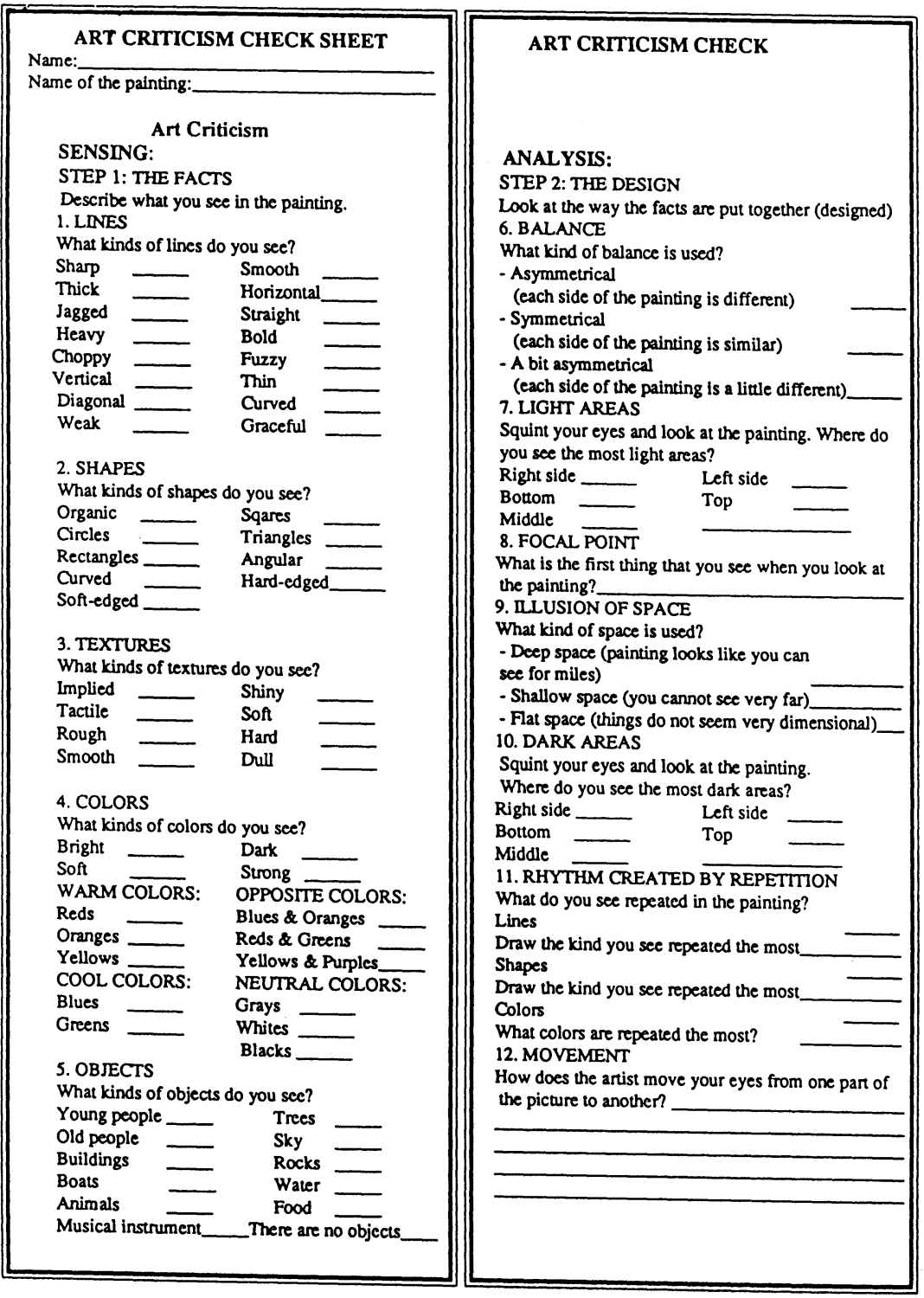




\section{ART CRITICISM CHECK SHEET}

\section{INTERPRETATION:}

\section{STEP 3: MEANING}

What is the purpose or meaning of the art work? Now, go back and reread how you described the facts (step 1) and how those facts are put together (step 2). These are the clues to the meaning and purpose of the painting. They will help you answer the following questions.

13. The artist seems to be primarily concerned with imitating nature.

$$
\text { Yes }
$$
No.

14. The artist seems to be mostly interested in expressing a feeling or an emotion. Yes__ No

15. The prime concern of this artist seems to be with lines, shapes, colors, and textures and with design or composition.

Yes.

No.

16. Does the name of the painting tell you about its meaning or purpose? Yes__ No

17. Which of the following words best describe what you think is the meaning of this art work (you may use as many words as you need and add some of your own):

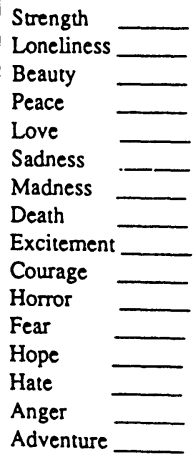

\section{ART CRITICISM CHECK SHEET}

\section{EVALUATION:}

STEP 4: JUDGMENT

Judge the painting.

18. This painting is an

excellent good bad

example of:

-imitationism (imitating nature)

-emotionalism (showing a feeling or emotion)

-formalism (making the viewer aware of lines, shapes, colors or design)

19. I like___ don't like___ this art work.

20. This work might be improved by

21. For the time or era in which this work was done this work is:

typical of this period

a new idea for the time

a modification of what had previously been done

an entirely new idea in art history

22. Responses

- This work makes me think about it

- This work stirs up strong feelings in me

- This work creates order

- This work seems complex

- This work causegme to wonder about 